\title{
Economical Evaluation of Runge-Kutta Formulae
}

\section{By David J. Fyfe}

1. (iill [1] and Blum [2] have produced special versions of the Runge-Kutta fourth order method for the solution of $N$ simultaneous first order differential equations which require $3 N+P$ storage locations against the normal $4 N+P$, where $P$ is the storage required by the program. It is shown below that it is possible to arrange all such methods in a form which requires $3 N+P$ storage locations. Gill's method for reducing round off error is also extended.

2. The Runge-Kutta fourth order methods for solving the $N$ equations

$$
\begin{aligned}
y_{i}{ }^{\prime} & =f_{i}\left(x, y_{1}, y_{2}, \cdots, y_{N}\right), \\
y_{i}\left(x_{0}\right) & =y_{i 0}, \quad i=1,2, \cdots, N,
\end{aligned}
$$

are usually written in the form $\left(y_{i j}=y_{i}\left(x_{j}\right)\right)$

where

$$
y_{i j+1}=y_{i j}+a k_{i 0}+b k_{i 1}+c k_{i 2}+d k_{i 3},
$$

$$
\begin{aligned}
k_{i 0}= & h f_{i}\left(x_{j}, y_{1 j}, y_{2 j}, \cdots, y_{N j}\right), \\
k_{i 1}= & h f_{i}\left(x_{j}+m h, y_{1 j}+m k_{10}, \cdots, y_{N j}+m k_{N 0}\right), \\
k_{i 2}= & h f_{i}\left(x_{j}+n h, y_{1 j}+(n-r) k_{10}+r k_{11}, \cdots, y_{N j}+(n-r) k_{N 0}+r k_{N 1}\right), \\
k_{i 3}= & h f_{i}\left(x_{j}+p h, y_{1 j}+(p-s-t) k_{10}+s k_{11}+t k_{12}, \cdots,+y_{N j}\right. \\
& \left.+(p-s-t) k_{N 0}+s k_{N 1}+t k_{N 2}\right), \\
& i=1,2, \cdots, N .
\end{aligned}
$$

The $a, b, c, d, m, n, p, r, s, t$ satisfy the following eight equations

$$
\begin{aligned}
a+b+c+d & =1, \\
b m+c n+d p & =\frac{1}{2}, \\
b m^{2}+c n^{2}+d p^{2} & =\frac{1}{3}, \\
c m r+d n t+d m s & =\frac{1}{6}, \\
b m^{3}+c n^{3}+d p^{3} & =\frac{1}{4}, \\
c m n r+d n t p+d m s p & =\frac{1}{8}, \\
c m^{2} r+d n^{2} t+d m^{2} s & =\frac{1}{12}, \\
d m r t & =\frac{1}{24} .
\end{aligned}
$$

The computation with the formulae arranged in this form requires $4 N+P$ storage

Received October 4, 1965. 
locations. It is now shown that by splitting the computation into four stages it is possible to obtain the solution by storing $3 N$ quantities at each stage.

Let

$$
\begin{aligned}
Z_{i 0} & =y_{i j}, \\
Z_{i 1} & =y_{i j}+m k_{i 0}, \\
Z_{i 2} & =y_{i j}+(n-r) k_{i 0}+r k_{i 1}, \\
Z_{i 3} & =y_{i j}+(p-s-t) k_{i 0}+s k_{i 1}+t k_{12}, \\
y_{i j+1} & \equiv Z_{i 4}=y_{i j}+a k_{i 0}+b k_{i 1}+c k_{i 2}+d k_{i 3} .
\end{aligned}
$$

Expressing each $Z_{i}$ in terms of the previous value we obtain

$$
\begin{aligned}
& Z_{i 0}=y_{i j}, \\
& Z_{i 1}=Z_{i 0}+m k_{i 0}, \\
& Z_{i 2}=Z_{i 1}+(n-m+r) k_{i 0}+r k_{i 1}, \\
& Z_{i 3}=Z_{i 2}+(p-s-t-n+r) k_{i 0}+(s-r) k_{i 1}+t k_{i 2}, \\
& Z_{i 4}=Z_{i 3}+(a-p+s+t) k_{i 0}+(b-s) k_{i 1}+(c-t) k_{i 2}+d k_{i 3} .
\end{aligned}
$$

Let $P_{i 0}=k_{i 0}=h f_{i}\left(x_{j}, Z_{10}, \cdots, Z_{N 0}\right)$, then $Z_{i 1}=Z_{i 0}+m P_{i 0}$.

Let $Q_{i 1}=P_{i 0}$, and $P_{i 1}=k_{i 1}=h f_{i}\left(x_{j}+m h, Z_{11}, \cdots, Z_{N 1}\right)$, then $Z_{i 2}=Z_{i 1}+$ $(n-m-r) Q_{i 1}+r P_{i 1}$.

The $P_{i 1}$ and $Z_{i 2}$ are stored in the locations occupied by $P_{i 0}$ and $Z_{i 1}$ as the latter are no longer required.

Let $Q_{i 2}=Q_{i 1}+A P_{i 1}$, and $P_{i 2}=k_{i 2}+B P_{i 1}$. If $A$ and $B$ are chosen so that

$$
(p-s-t-n+r) A+t B=(s-r),
$$

then $Z_{i 3}=Z_{i 2}+(p-s-t-n+r) Q_{i 2}+t P_{i 2}$. Again the new $P_{i}, Q_{i}, Z_{i}$ replace the previous triplet.

Let $Q_{i 3}=Q_{i 2}$, and $P_{i 3}=k_{i 3}+C P_{i 2}$. If $A, B, C$ are chosen so that

$$
(a-p+s+t) A+d B C=(b-s)
$$

and

$$
d C=(c-t)
$$

then $Z_{i 4}=Z_{i 3}+(a-p+s+t) Q_{i 3}+d P_{i 3}$. In the above equations each triplet $Z, P, Q$ is expressed in terms of the previous triplet only and so only $3 N$ storage locations are required.

Solving (3), (4) and (5) we obtain

$$
\begin{aligned}
A & =\frac{(c-t)(s-r)-t(b-s)}{(c-t)(p-s-t-n+r)-t(a-p+s+t)}, \\
B & =\frac{s-r}{t}-\frac{(p-s-t-n+r)}{t} A, \\
C & =\frac{c-t}{d},
\end{aligned}
$$

( $d=0$ or $t=0$ is impossible from (2)). 
Thus if the equations are arranged as follows only $3 N+P^{P}$ storage locations are required:

$$
\begin{aligned}
Z_{i 0} & =y_{i j} \\
P_{i 0} & =h f_{i}\left(x_{j}, Z_{10}, \cdots, Z_{N 0}\right) \\
Z_{i 1} & =Z_{i 0}+n P_{i 0} \\
Q_{i 1} & =P_{i 0} \\
P_{i 1} & =h f_{i}\left(x_{j}+m h, Z_{11}, \cdots, Z_{N 1}\right) \\
Z_{i 2} & =Z_{i 1}+(n-m-r) Q_{i 1}+r P_{i 1} \\
Q_{i 2} & =Q_{i 1}+A P_{i 1}, \\
P_{i 2} & =h f_{i}\left(x_{j}+n h, Z_{12}, \cdots, Z_{N 2}\right)+B P_{i 1} \\
Z_{i 3} & =Z_{i 2}+(p-s-t-n+r) Q_{i 2}+t P_{i 2} \\
Q_{i 3} & =Q_{i 2} \\
P_{i 3} & =h f_{i}\left(x_{j}+p h, Z_{13}, \cdots, Z_{N 3}\right)+C P_{i 2} \\
y_{i j+1} & \equiv Z_{i 4}=Z_{i 3}+(a-p+s+t) Q_{i 3}+d P_{i 3} .
\end{aligned}
$$

3. Gill's method of reducing round-off error is now applied to Equations (6). In this method artificial round-off errors are introduced to minimize the actual round-off error. This is possible because the quantities $P_{i}$ and $Q_{i}$ are of order $h$ and so in general can be stored to a higher degree of accuracy than the $Z_{i}$. This is done automatically if floating point arithmetic is used. In the first equation of each triplet in Equations (6) (i.e. the calculation of $Z_{i}$ ) we are concerned with adding quantities of order $h$ to quantities of order 1 . We will show below that, by introducing appropriate modifications in the terms of order $h\left(Q_{i}\right)$, it is possible to compensate almost exactly for the errors in $Z_{i}$. If we let the round-off error in the calculation of $P_{i 0}, Z_{i 1}, \cdots$, be $e\left(P_{i 0}\right), e\left(Z_{i 1}\right), \cdots$, which we suppose are easily available when these quantities are computed, the total round-off error accumulated in one step is

$$
\begin{aligned}
& E_{i}=e\left(Z_{i 1}\right)+e\left(Z_{i 2}\right)+e\left(Z_{i 3}\right)+e\left(Z_{i 4}\right) \\
& \quad+a e\left(P_{i 0}\right)+b e\left(P_{i 1}\right)+c e\left(P_{i 2}\right)+d e\left(P_{i 3}\right) \\
& \quad+(a-m) e\left(Q_{i 1}\right)+(a-n+r) e\left(Q_{i 2}\right)+(a-p+s+t) e\left(Q_{i 3}\right) .
\end{aligned}
$$

We now introduce modifications $e^{\prime}\left(Q_{i}\right)$ in the $Q_{i}$ to compensate almost exactly for the errors in $Z_{i}$ i.e. put

$$
\begin{aligned}
(a-m) e^{\prime}\left(Q_{i 1}\right) & =-e\left(Z_{i 1}\right), \\
(a-n+r) e^{\prime}\left(Q_{i 2}\right) & =-e\left(Z_{i 2}\right), \\
(a-p+s+t) e^{\prime}\left(Q_{i 3}\right) & =-e\left(Z_{i 3}\right) .
\end{aligned}
$$

In order to introduce these modifications we define quantities $R_{i 1}, R_{i 2}, R_{i 3}$ as 
follows:

$$
\begin{aligned}
& R_{i 1}=Z_{i 1}-Z_{i 0}=m P_{i 0}+e\left(R_{i 1}\right) \\
& R_{i 2}=Z_{i 2}-Z_{i 1}=(n-m-r) Q_{i 1}+r P_{i 1}+e\left(R_{i 2}\right), \\
& R_{i 3}=Z_{i 3}-Z_{i 2}=(p-s-t-n+r) Q_{i 2}+t P_{i 2}+e\left(R_{i 3}\right),
\end{aligned}
$$

where $e\left(R_{i j}\right)=e\left(Z_{i j}\right), j=1,2,3$.

We thus introduce $e^{\prime}\left(Q_{i 1}\right), e^{\prime}\left(Q_{i 2}\right), e^{\prime}\left(Q_{i 3}\right)$ such that

$$
\begin{aligned}
(a-m) e^{\prime}\left(Q_{i 1}\right) & =-e\left(R_{i 1}\right)=-\left(R_{i 1}-m P_{i 0}\right), \\
(a-n+r) e^{\prime}\left(Q_{i 2}\right) & =-e\left(R_{i 2}\right)=-\left(R_{i 2}-(n-m-r) Q_{i 1}-r P_{i 1}\right), \\
(a-p+s+t) e^{\prime}\left(Q_{i 3}\right) & =-e\left(R_{i 3}\right)=-\left(R_{i 3}-(p-s-t-n+r) Q_{i 2}-t P_{i 2}\right)
\end{aligned}
$$

which almost exactly compensate for the errors in $Z_{i 1}, Z_{i 2}, Z_{i 3}$. Therefore, redefine $Q_{i 1}, Q_{i 2}, Q_{i 3}$ as follows: $Q_{i j}^{\prime}=Q_{i j}+e^{\prime}\left(Q_{i j}\right), j=1,2,3$.

$$
\begin{aligned}
Q_{i 1}^{\prime} & =\left(\frac{a}{a-m}\right) P_{i 0}-\frac{1}{a-m} R_{i 1} \\
Q_{i 2}^{\prime} & =\left(\frac{a-m}{a-n+r}\right) Q_{i 1}^{\prime}+\left(A+\frac{r}{a-n+r}\right) P_{i 1}-\frac{1}{a-n+r} R_{i 2}, \\
Q_{i 3}^{\prime} & =\left(\frac{a-n+r}{a-p+s+t}\right) Q_{i 2}^{\prime}+\frac{t}{a-p+s+t} P_{i 2}-\frac{1}{a-p+s+t} R_{i 3} .
\end{aligned}
$$

Making the above change requires only one additional storage location as the $R_{i}$ 's are used temporarily in the formation of the $Z_{i}$ and $Q_{i}{ }^{\prime}$. The primes on $Q_{i}$ will now be dropped for convenience. The only large error term remaining is $e\left(Z_{i 4}\right)$. In order to eliminate this introduce $Q_{i 0}, R_{i 4}$ and $Q_{i 4}$, where $Q_{i 4}$ at one step becomes $Q_{i 0}$ at the next.

Define:

$$
\begin{aligned}
& R_{i 4}=Z_{i 4}-Z_{i 3}=(a-p+s+t) Q_{i 3}+d P_{i 3}+e\left(R_{i 4}\right) \\
& Q_{i 4}=e\left(R_{i 4}\right)=R_{i 4}-(a-p+s+t) Q_{i 3}-d P_{i 3} \\
& Q_{i 0}=\left[Q_{i 4}\right]_{x=x_{j}} .
\end{aligned}
$$

$Q_{i 4}$ is the round-off error in $Z_{i 4}$.

Since $Z_{i 0}=y_{i j}$, the best available estimate would appear to be $\left[Z_{i 4}-Q_{i 4}\right]_{x=x_{j}}$ which gives

$$
R_{i 1}=Z_{i 1}-y_{i j}=Z_{i 1}-Z_{i 0}+Q_{i 0} .
$$

Thus we redefine $R_{i 1}$ and consequently $Q_{i 1}$ as follows:

$$
R_{i 1}=m P_{i 0}-Q_{i 0}+e\left(R_{i 1}\right)
$$

and

$$
Q_{i 1}=\left(\frac{a}{a-m}\right) P_{i 0}-\frac{1}{a-m} Q_{i 0}-\frac{1}{a-m} R_{i 1} .
$$

However, it will now be shown, following Gill [1], that it is slightly better to let 


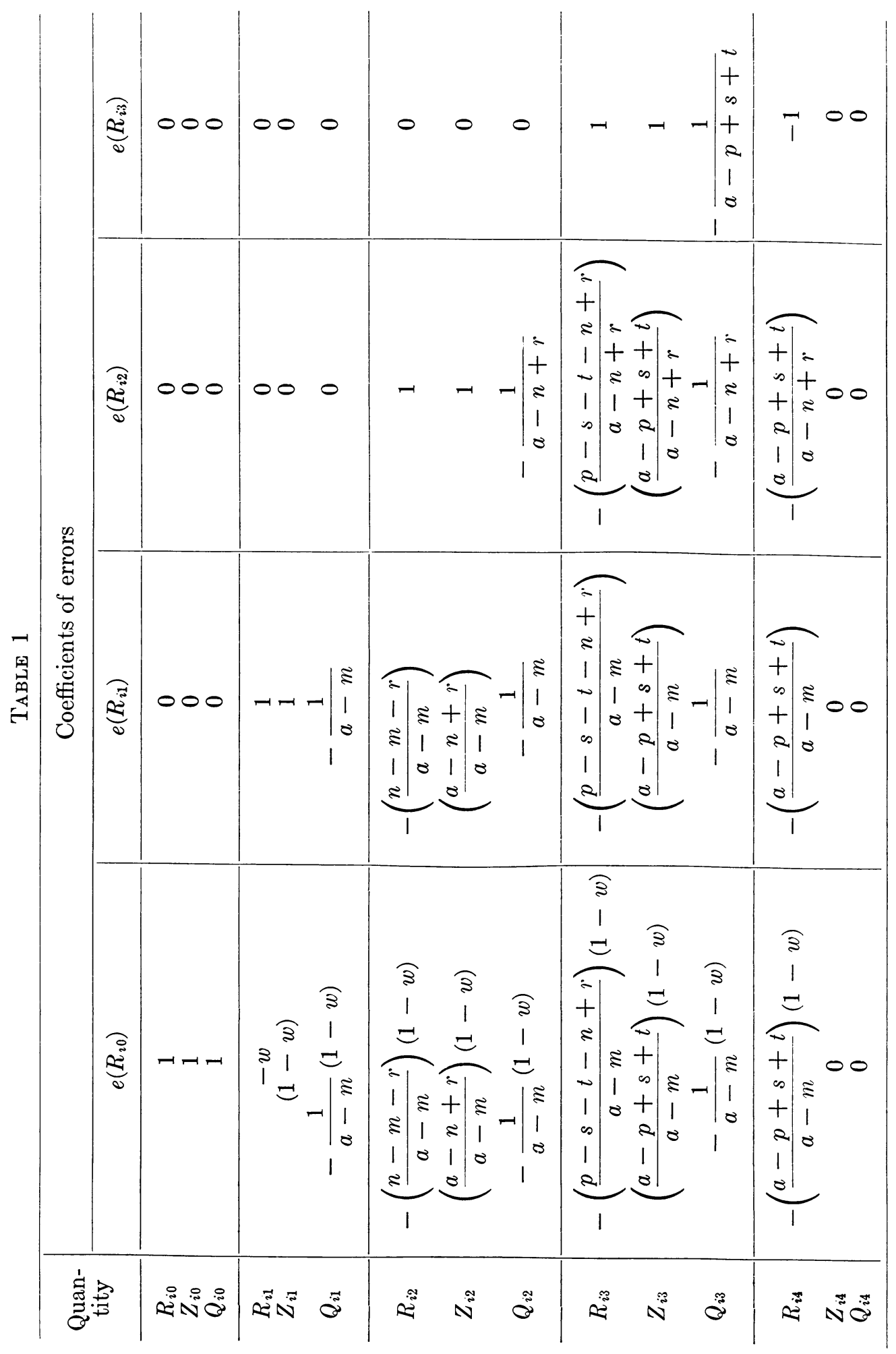




$$
R_{i 1}=m P_{i 0}-w Q_{i 0}+e\left(R_{i 1}\right)
$$

where

$$
w=1+\frac{2 a(a-m)}{2 a(1-a)-1+2(c r+d s+d t)} .
$$

Table 1 shows the errors in each quantity $R_{i}, Z_{i}, Q_{i}$ within one step of errors $e\left(R_{i 0}\right), e\left(R_{i 1}\right), e\left(R_{i 2}\right), e\left(R_{i 3}\right)$, where $R_{i 0}=\left[R_{i 4}\right]_{x=x_{j}}$. The error in $y_{i j+1}$ due to errors in the $P_{i}$ caused by errors in $Z_{i}$ is given by

$$
E\left(y_{i j+1}\right)=a E\left(P_{i 0}\right)+b E\left(P_{i 1}\right)+c E\left(P_{i 2}\right)+d E\left(P_{i 3}\right)
$$

where $E\left(P_{i 0}\right)$ represents the total error in $P_{i 0}$ etc. If we assume that the partial derivatives $\partial f_{i} / \partial y_{k}, k=1,2, \cdots, N$, are constant over one step

$$
\begin{aligned}
E\left(y_{i \jmath+1}\right) & =h \sum_{k=1}^{N} \frac{\partial f_{i}}{\partial y_{k}}\left\{a E\left(Z_{k 0}\right)+b E\left(Z_{k 1}\right)+c E\left(Z_{k 2}\right)+d E\left(Z_{k 3}\right)\right\}, \\
& =h \sum_{k=1}^{N} \frac{\partial f_{i}}{\partial y_{k}}\left\{\operatorname{Se}\left(R_{k 0}\right)+T e\left(R_{k 1}\right)+U e\left(R_{k 2}\right)+\operatorname{Ve}\left(R_{k 3}\right)\right\},
\end{aligned}
$$

where

$$
\begin{aligned}
& S=a+(1-w)\left[b+c\left(\frac{a-n+r}{a-m}\right)+d\left(\frac{a-p+s+t}{a-m}\right)\right] \\
& T=b+c\left(\frac{a-n+r}{a-m}\right)+d\left(\frac{a-p+s+t}{a-m}\right) \\
& U=c+d\left(\frac{a-p+s+t}{a-n+r}\right) \\
& V=d .
\end{aligned}
$$

Assuming the $e\left(R_{i}\right)$ are randomly distributed between $-\frac{1}{2}$ unit and $+\frac{1}{2}$ unit, the standard deviation in $y_{i j+1}$ from this source is a minimum if $S=0$, which leads to the optimum value

$$
\begin{aligned}
w & =1+\frac{a(a-m)}{b(a-m)+c(a-n+r)+d(a-p+s+t)}, \\
& =1+\frac{2 a(a-m)}{2 a(1-a)-1+2(c r+d s+d t)} \quad \text { (from Equation (2)). }
\end{aligned}
$$

The final formulae are therefore:

$$
\begin{aligned}
Z_{i 0} & =y_{i j}, \quad Q_{i 0}=\left[Q_{i 4}\right]_{x=x_{j}}, \\
P_{i 0} & =h f_{i}\left(x_{j}, Z_{10}, \cdots, Z_{N 0}\right) \\
R_{i 1} & =m P_{i 0}-w Q_{i 0}+e\left(R_{i 1}\right) \\
Z_{i 1} & =Z_{i 0}+R_{i 1} \\
Q_{i 1} & =\left(\frac{a}{a-m}\right) P_{i 0}-\frac{1}{a-m} Q_{i 0}-\frac{1}{a-m} R_{i 1}, \\
P_{i 1} & =h f_{i}\left(x_{j}+m h, Z_{11}, \cdots, Z_{N 1}\right)
\end{aligned}
$$




$$
\begin{aligned}
R_{i 2} & =(n-m-r) Q_{i 1}+r P_{i 1}+e\left(R_{i 2}\right) \\
Z_{i 2} & =Z_{i 1}+R_{i 2}, \\
Q_{i 2} & =\left(\frac{a-m}{a-n+r}\right) Q_{i 1}+\left(A+\frac{r}{a-n+r}\right) P_{i 1}-\left(\frac{1}{a-n+r}\right) R_{i 2}, \\
P_{i 2} & =h f_{i}\left(x_{j}+n h, Z_{12}, \cdots, Z_{N 2}\right)+B P_{i 1} ; \\
R_{i 3} & =(p-s-t-n+r) Q_{i 2}+t P_{i 2}+e\left(R_{i 3}\right), \\
Z_{i 3} & =Z_{i 2}+R_{i 3}, \\
Q_{i 3} & =\left(\frac{a-n+r}{a-p+s+t}\right) Q_{i 2}+\left(\frac{t}{a-p+s+t}\right) P_{i 2}-\frac{1}{a-p+s+t} R_{i 3}, \\
P_{i 3} & =h f_{i}\left(x_{j}+p h, Z_{13}, \cdots, Z_{N 3}\right)+\frac{c-t}{d} P_{i 2} ; \\
R_{i 4} & =(a-p+s+t) Q_{i 3}+d P_{i 3}+e\left(R_{i 4}\right) \\
y_{i j+1} & \equiv Z_{i 4}=Z_{i 3}+R_{i 4}, \\
Q_{i 4} & =R_{i 4}-(a-p+s+t) Q_{i 3}-d P_{i 3} .
\end{aligned}
$$

Mathematics Department

Woolwich Polytechnic

London, England

1. S. Gill, "A process for the step-by-step integration of differential equations in an automatic digital computing machine," Proc. Cambridge Philos. Soc., v. 47, 1951, pp. 95-108. MR 12, 538.

2. E. K. Burm, "A modification of the Runge-Kutta fourth order method," Math. of Comp., v. 16, 1962, pp. 176-187. MR $26 \# 3190$. 